Instructions for authors, subscriptions and further details:

http://rasp.hipatiapress.com

\title{
Materia Impresa: Una Investigación desde la Práctica Artística.
}

Marta Negre ${ }^{1}$

1) Facultad de Bellas Artes. Universidad de Barcelona. España

Date of publication: June 3rd, 2018

Edition period: February 2018 - June 2018

To cite this article: Negre, Marta (2018). Materia Impresa: una Investigación desde la Práctica Artística. (Recensión del libro Materia Impresa: De la Virtualidad Digital a la Tridimensionalidad en la Investigación Artística). Barcelona, Research, Art, Creation, 6(2), 228 - 230. doi: 10.17583/brac.2018.2952

To link this article: http://dx.doi.org/10.17583/brac.2018.2952

\section{PLEASE SCROLL DOWN FOR ARTICLE}

The terms and conditions of use are related to the Open Journal System and to Creative Commons Attribution License (CC-BY). 
BRAC-Barcelona Research Art Creation Vol. 6 No. 2 June 2018 pp. 228-230

(Received: 8 September 2017; Accepted: 24 May 2018; Published: 3 June 2018)

\section{Review}

V.V.A.A. (2017). Materia impresa: de la virtualidad digital a la tridimensionalidad en la investigación artística. Barcelona: Comanegra.

Materia impresa, publicación del equipo integrante del proyecto homónimo de investigación $\mathrm{I}+\mathrm{D}$, reúne un conjunto de artículos y propuestas artísticas analizando desde puntos de vista y metodologías diversas la aplicación de la impresión 3D en las artes, más allá de la experimentación plástica, planteando así el modo en el que esta tecnología es capaz de proporcionar una nueva manera de entender la escultura y, por extensión, los lenguajes artísticos. Estos avances técnicos han tenido siempre una gran incidencia en las producciones artísticas generando grandes cambios. Alguno fruto de las necesidades creativas del momento i otros-de mayor proyección-generando giros importantes en su concepción. Desde esta conciencia, el proyecto Materia impresa tiene como objetivo averiguar qué significa trabajar con tecnología 3D y cómo los artistas afrontan este procedimiento. El libro muestra parte de la producción llevada a cabo por el propio equipo, con reflexiones teóricas relacionadas con el quehacer productivo, partiendo de la premisa que la investigación en arte no puede disociarse de su práctica: es desde ésta que se visualiza la complejidad de cada uno de los aspectos. 
Concretamente, en el libro se desarrollan, entre otras, tres ideas interesantes de resaltar. En primer lugar, Albert Valera nos habla, citando a Chris Anderson, de la necesidad de volver a la fisicidad del material. Es decir, se detecta una voluntad en el ámbito creativo de que las piezas superen su proyecto virtual, para conseguir tomar forma y presencia más allá de la pantalla. Esta materialidad se observa en muchas de las propuestas artísticas de los investigadores, ya que la mayoría tienen un gran componente táctil. En segundo lugar, se analiza el cambio de paradigma que supone idear un objeto tridimensional a partir de claves numéricas, sea éste artístico o no. En este sentido, Ariadna Fàbregas, autora de uno de los artículos, ve la tecnología 3D como una herramienta que puede utilizarse de tres maneras: como instrumento que describe el proyecto, como instrumento que lo performa o como instrumento transformador de la propia tecnología. Finalmente, otro concepto crucial y que se constata en todo el libro es la tendencia hacia lo interdisciplinar y al trabajo en equipo. En realidad, es lógico que en el terreno de la impresión 3D arte y ciencia vayan de la mano, con el fin de nutrirse mutuamente y de ampliar las posibilidades de cada uno de los dos ámbitos.

En cuanto a los contenidos, el ejemplar se estructura en tres apartados: «Tecnología 3D versus Arte», «Proyectos» e «Investigación artística y avances tecnológicos». En el primero se analiza esta técnica y su repercusión en el arte. Para hacerlo se incluyen tres ensayos, empezando con el artículo de Ariadna Fàbregas, en el cual muestra como el hardware, el software y el dibujo paramétrico condicionan el proceso de proyección. A continuación, Salvador Juanpere presenta artistas contemporáneos que se han servido de esta tecnología como mecanismo útil para materializar unas obras que dialogan con temas sociales y políticos. Después, Francisco Javier Lozano se centra en la relación entre arte y ciencia. El autor hace un recorrido histórico por diferentes herramientas digitales, la evolución de la impresión 3D y su impacto en el arte. A la vez, introduce ideas que han surgido de este contexto, como el código abierto o el procomún colaborativo, nociones que han favorecido la aparición de nuevas formas de relación y comunidades virtuales.

En el segundo apartado se publican cinco proyectos elaborados a partir de las inquietudes artísticas y conceptuales de los investigadores. El 
resultado es un conjunto de piezas que indagan en las posibilidades de la impresión 3D, a la vez que reflexionan acerca de sus límites y sus connotaciones léxicas. Por ejemplo, Mercè Casanovas, con la reproducción mimética de un fósil de pez, plantea una relación ambigua entre realidad y ficción. También desde el binomio original y copia, Salvador Juanpere examina las capacidades del medio. En su propuesta extrae, como si fuera la epidermis, el contorno de la emblemática Musa de Brancusi. Por su parte, Cristina Pastó, enlazando arte y ciencia, construye unas réplicas de líquenes imprimiendo cinco módulos que se superponen, los cuales forman una estructura orgánica similar a la natural. Aproximándose a la arquitectura, Albert Valera realiza una pieza escultórica que remite al límite real donde se unen y se separan los edificios. Con ello hace referencia a los puntos de encuentro que se propician en el entorno urbano. Finalmente, Àngels Viladomiu produce lo que llama un souvenir de una cordillera, sugiriendo una mirada crítica hacia la relación que se instaura con el paisaje a partir de la incursión de las nuevas tecnologías.

Para terminar, el último apartado, «Investigación artística y avances tecnológicos», está constituido por cuatro ensayos, en los que Àngels Viladomiu, Jordi Bielsa, Francisco Javier Lozano y Enrique Martínez Leal especulan acerca de sus proyectos artísticos. En sus observaciones se pone de manifiesto que la práctica artística funciona como proceso de investigación, y que la producción de obra es inherente al corpus conceptual que la sostiene y determina.

En definitiva, este libro contiene interesantes consideraciones tanto teóricas como prácticas, y se erige como un estudio oportuno sobre una tecnología que supondrá un cambio en la manera de trabajar y entender lo tridimensional, así como en la forma de fabricar objetos y relacionarnos con ellos, no solo en lo que al arte se refiere. 